\title{
Is helmet CPAP first line pre-hospital treatment of Presumed Severe
}

Acute Pulmonary Oedema?

Giuseppe Foti $^{1}$ MD, Fabio Sangalli ${ }^{1}$ MD, Lorenzo Berra ${ }^{1}$ MD, Stefano Sironi ${ }^{2}$ RN, Marco Cazzaniga $^{2}$ MD, Giampiera Rossi ${ }^{2}$ MD, Giacomo Bellani ${ }^{1,3}$ MD and Antonio Pesenti MD ${ }^{1,3}$.

1 Department of Perioperative Medicine and Intensive Care, San Gerardo Hospital, Monza (MI), Italy

2 Department of Perioperative Medicine and Intensive Care - Servizio Sanitario di Urgenza ed Emergenza Medica (SSUEm)-Brianza, San Gerardo Hospital,Monza (MI), Italy

3 Department of Experimental Medicine, University of Milan-Bicocca, Monza (MI), Italy.

Corresponding author: Giuseppe Foti, MD

Department of Perioperative Medicine and Intensive Care

San Gerardo Hospital,

Via Pergolesi 33

20052 Monza (MI), Italy

\section{Funding source}

All authors received a salary from their respective institutions. No additional funding was received for the study 
Abstract

PURPOSE: Non invasive Continuous Positive Airway Pressure (CPAP) is effective in reducing intubation rate and mortality of patient with Acute Cardiogenic Pulmonary Edema (ACPE). We report our experience on pre-hospital application of CPAP by helmet as an adjunct to medical therapy or as a stand alone procedure in patient with presumed ACPE. METHODS: In pre-hospital treatment of 62 patients with presumed ACPE, CPAP was added to standard medical treatment while in another 59 patients, CPAP was used as a sole therapy . RESULTS: Helmet CPAP was feasible in all patients. No patient required pre-hospital intubation. In both groups, CPAP significantly improved oxygenation $\left(\mathrm{SpO}_{2}\right.$ went from $79 \pm 12 \%$ to $97 \pm 3 \%$ and from $81 \pm 13 \%$ to $98 \pm 3 \%$ ), reduced respiratory rate (from $26 \pm 4$ to $21 \pm 3 \mathrm{bpm}$ and from $30 \pm 9$ to $22 \pm 8 \mathrm{bpm}$ ) and improved hemodynamics, with a more pronounced decrease in blood pressure in the group with medical treatment than in the one without it. In the two cohorts, four and five patients were respectively intubated in Emergency Department and eleven and nine eventually died.

CONCLUSIONS: Helmet CPAP is feasible, efficient and safe in pre-hospital treatment of presumed ACPE. A significant improvement of physiological variables was observed also in the group treated with CPAP in absence of a drug therapy. We propose helmet CPAP as first line prehospital treatment of presumed severe ACPE.

Keywords: Pulmonary Oedema, Positive Pressure Ventilation, Pre-Hospital, CPAP 


\section{Introduction}

Respiratory distress is the second most common symptom of adults transported by ambulance and acute cardiogenic pulmonary oedema (ACPE) is one of the leading causes of respiratory distress in cardiac patients [1]. In hospital, non-invasive Continuous Positive Airway pressure (CPAP) has proven effective in ameliorating gas exchange and reducing both the need for endotracheal intubation and the overall mortality of patients with ACPE, as suggested by randomized controlled trials and a recent meta-analysis [2-10], although this result has been challenged by a large multicenter trial. [11]

With the understanding that immediate advanced medical care is essential to improve the long-term outcome of cardiac patients, we developed a program for the pre-hospital application of CPAP. A similar approach to patients with ACPE has been recently described by Plaisance and coworkers [12], The study showed that CPAP alone was more effective than medical treatment alone in improving respiratory function. Moreover, a delayed application of CPAP appeared to be associated with an increased intra-hospital mortality.

CPAP is commonly applied through face masks requiring a tight seal to the patient's face to prevent air leaks and loss of pressure. The application of a face mask requires patient cooperation, an experienced team, and either a reservoir chamber or a high flow gas source to achieve a constant airway pressure and inspired oxygen fraction. Moreover face masks are sometimes impossible to use due to peculiar patient's characteristics (e.g. in an edentulous patient, or in those wearing a beard); they are sometimes uncomfortable or poorly tolerated, possibly limiting the duration of application [13-15]. The correct application of face masks is therefore somehow difficult even in an Intensive Care Unit (ICU) or in the Emergency Room (ER), and it may be very problematic or ineffective in the pre-hospital setting. 
We therefore selected the helmet for prehospital CPAP application: it appears to be as effective as face mask [16], easily applied, well tolerated and clinically beneficial [14, 17, 18].

We report here our experience and observations about the pre-hospital application of helmet CPAP in patient with presumed ACPE. We will discuss:

- the feasibility, safety, and efficacy of helmet CPAP

- the effects of helmet CPAP as an addition to standard medical therapy or as a stand alone treatment.

\section{Methods}

\section{The Emergency Medical System (EMS) of Monza-Brianza}

This study was approved by the institutional review board for collecting data from the patient's medical records. The study was conducted in Brianza, a region located in Lombardia, Italy. All EMS activities are co-ordinated by a local operative center (SSUEM) dispatches a Basic Life Support, and in selected cases (including reported respiratory distress) an Advanced Life Support (ALS) team.

Basic Life Support teams are staffed by three specifically trained lay volunteers; ALS teams can be staffed either with a technician, a nurse and a physician (ALSdoc) or with two volunteers and a nurse (ALSnurs). According to the Italian Legislation at the time of the study, ALSnurs might only perform limited therapeutic interventions and no drug administration. ALSdoc and ALSnurs teams cover different areas and are dispatched according solely to the site of origin of the call.

All the ALS crews underwent a 4 hours training course on the use of the helmet device. 


\section{Patients}

We enrolled patients with suspected ACPE rescued by ALSdoc between December 1, 1998 and December 31, 1999 and patients treated by ALSnurs between February 1, 2001 and December 1, 2002.

\section{Protocol}

Inclusion and exclusion criteria are reported in Table 1.

The diagnosis of suspected ACPE was based on medical history positive for cardiovascular disease and physical examination including orthopnea, diffuse crackles without evidence of pulmonary aspiration of infection, and pulse oximetry. In the ALSnurs group the diagnosis was always validated on the phone by the physician in charge at the SSUEM. In the ALSdoc group, the severity of ACPE was also assessed by means of a "Wet Rales Score" (WRS), ranging from 1 to 4, based on the extension of crackles and/or rales evaluated at chest auscultation.

Upon arrival, the ALS team administered supplemental oxygen via a bag mask at $15 \mathrm{~L} / \mathrm{min}$, secured a venous line and (in the ALSdoc group) pharmacological therapy was started according to ACLS guidelines. ALSnurs avoided drug administration. In the vast majority of cases oxygen administration had been started by Basic Life Support crews before ALS arrival. Respiratory rate, heart rate, non-invasive arterial pressure, pulse oximetry, and WRS were recorded. If the protocol criteria were met, CPAP was applied while medical therapy was continued as indicated. Vital parameters were monitored throughout all transportation period and the final reading upon arrival in the ER was recorded and used for data analysis.

Helmet CPAP was discontinued in case of a worsening level of consciousness, shock, intolerance or whenever endotracheal intubation was required.

In the ER, CPAP was continued as clinically indicated and the hypothesis of ACPE was confirmed after routine diagnostics, including chest x-ray. 
We also recorded the need for pre-hospital or in-hospital endotracheal intubation, type of admission (ICU or general ward), length of hospital stay, outcome at discharge.

\section{Helmet-CPAP administration}

A detailed description of the helmet CPAP system is provided elsewhere [16, 19]. Basically, the helmet consists of an oxygen head tent (Sea Long Med. Sys Inc, Louisville, USA) with two side ports. The inlet port was connected to a high-flow oxygen source while the outlet port was connected to a mechanical spring PEEP valve (PEEP-FLOW, Bird, Dallas, USA). During the onsite application, transport to the ambulance and transfer from the ambulance to the ER, the inlet port was connected to a portable oxygen cylinder (3 L capacity filled at 200 Atmospheres); the ambulance oxygen supply was used while on board. An oxygen flow of at least $30 \mathrm{~L} / \mathrm{min}$ was used to prevent $\mathrm{CO}_{2}$ rebreathing [16]; the presence of a continuous flow from the PEEP valve throughout all the respiratory cycle was used as an indication of an adequate pneumatic circuit performance. ALSdoc could apply PEEP levels between 5 and $15 \mathrm{~cm} \mathrm{cmH}_{2} \mathrm{O}$ according to oximetry and clinical response, while ALSnurs maximum PEEP level was $10 \mathrm{cmH}_{2} \mathrm{O}$. Elastic straps were positioned under the patient's armpits and fixed to the helmet.

\section{End-points}

Primary endpoints of this study were the assessment of the feasibility of helmet CPAP in the outhospital setting and its efficacy, defined as improvement in the recorded clinical variables. Secondary endpoint was to compare the efficacy of CPAP when administered as a sole treatment or in conjunction with standard medical therapy.

\section{Statistical analysis}

Values are expressed as mean \pm SD. Changes observed following CPAP administration were compared using paired Student's $t$-test and Wilcoxon-signed rank test when appropriate. 
Differences among ALSdoc and ALSnurs groups were evaluated by unpaired Student's $t$-test and chi-square test when appropriate.

$\mathrm{P}<0,05$ were considered as statistically significant.

\section{Results}

\section{General Results}

We enrolled 62 consecutive patients rescued by ALSdoc and 59 consecutive patients rescued by ALSnurs. Patients' characteristics and main outcome results are summarized in Table 2.

The diagnosis of ACPE was confirmed in hospital in 104 out of 121 patients (86\%). Of the 17 non confirmed ACPE diagnosis, eight patients were diagnosed with pneumonia, five with malignancies and four with ARDS. Twenty-two patients were admitted to an ICU, 91 were transferred to a general ward, while eight died during the first $24 \mathrm{hrs}$ in the ER. The overall hospital stay was $10 \pm$ 8.3 days and the hospital mortality was $16.6 \%$ (20 out of 121 patients).

No patients required a pre hospital endotracheal intubation, while nine patients were intubated in hospital. Six of this patients had been diagnosed as non ACPE in the ER. One patient per group was enclosed in the protocol although the recorded SBP was between 80 and $90 \mathrm{mmHg}$. Vasoactive drugs employed by ALSdoc group are summarized in Table 2. 56 out of 62 patients underwent drugs therapy while in six patients, a venous line was not available. No drugs were administered in ALSnurs.

\section{Feasibility and efficacy of Helmet CPAP}

Helmet CPAP was applied and tolerated for all the pre-hospital phase in all the 121 patients. The application of CPAP did not lead to any adverse event directly related to positive pressure (gastric 
distension, aspiration of gastric content, pneumothorax), or to helmet use (skin lesions to the neck due to the rubber base, claustrophobia or discomfort).

The PEEP level applied was $10.6 \pm 1.7$ and $7.4 \pm 2.3 \mathrm{cmH}_{2} \mathrm{O}(\mathrm{p}<0.01)$ in ALSdoc and ALSnurs respectively.

Selected respiratory and circulatory function data are summarized in Table 3. In both groups, following CPAP administration pulse oximetry values normalized. Respiratory rate and, when available, WRS score decreased toward normality. Heart rate decreased significantly in both groups and Systolic Blood Pressure (SBP) also decreased significantly during CPAP application. Interestingly this was true also in the ALSnurs group in which no pharmacological treatment was applied.

In Figure 1 relative changes of SBP following helmet CPAP are plotted as a function of their initial values while breathing oxygen in the bag mask. Both groups showed a significant linear inverse relationship, indicating that the higher the initial SBP the larger will be its reduction after therapy; however for the lowest values of initial SBP there is no further tendency to a reduction in blood pressure but, on the contrary, a reverse trend toward improvement in blood pressure was observed.

\section{Comparison of Helmet CPAP with and without pharmacologic treatment}

The baseline data of ALSdoc and ALSnurs were comparable, except for basal respiratory rate, which was higher in the ALSnurs group; a similar proportion of ACPE diagnosis were confirmed ( $87 \%$ in the ALSdoc and $85 \%$ in the ALSnurs, $p=n s)$. Nine patients $(14.5 \%)$ in ALSdoc and 13 (22 $\%)$ in ALSnurs were admitted to an ICU ( $\mathrm{p}=\mathrm{ns})$. When only patients with a confirmed ACPE diagnosis are considered, the mortality amounted to $7.4 \%$ (4 out 54) and $10 \%$ (5 out of 50) in the ALSdoc and ALSnurs groups respectively $(\mathrm{p}=\mathrm{ns})$.

No difference was recorded in the hospital stay averaging $9.2 \pm 7.9$ and $10.8 \pm 8.8$ days ( $\mathrm{p}=\mathrm{ns}$ ) in the ALSdoc and ALSnurs group respectively. 
On the right hand-side of Table 3, relative variations of the selected parameters are displayed: magnitude of variation was not different for all the variables, except for SBP that decreased more in the ALSdoc group $(-14 \pm 13 \%$ vs $-7 \pm 15 \% ; \mathrm{p}<0 \cdot 01)$.

\section{Discussion}

The main results of the present study are that helmet CPAP is feasible and can be safely and easily applied in the pre hospital setting to patients with a presumed diagnosis of ACPE. Its application is associated to a prompt and sustained improvement of the respiratory and circulatory function, even in absence of any specific pharmacological intervention (ALSnurs group).

\section{Limitations of the study}

Since patients were not randomized to treatment with or without helmet CPAP, one might speculate that the improvement in clinical parameters was due to medical therapy. If this were the case, however, the improvement should have been larger in the ALSdoc than in the ALSnurs group. This was not the case except for a more pronounced reduction in SBP in ALSdoc while all the other vital parameters monitored improved similarly in both groups There was also no randomization between ALSdoc and ALSnurs teams, since not feasible, as the teams cover different geographic areas: this should, however, minimize the selection bias, since it was impossible to decide, based on patients condition, which team to send. Moreover neither patients nor rescue teams were blinded to the treatment being administered.

\section{Out of hospital use of CPAP}

The efficacy of CPAP in ACPE is well established. [2-10]. Despite this background, reports of CPAP application in prehospital setting are, to our knowledge quite limited. Kosowsky et al [20], reported the ambulance use of a high flow Venturi mask CPAP system and Templier et al.[21], that 
reported about 50 patients treated by Boussignac CPAP on their way to the hospital. In both studies CPAP was an adjunct to the standard drug protocol; both studies suggested a wider use of CPAP. More recently Plaisance et al.[12] reported the use of out of hospital CPAP in patients with ACPE: this study shares some similarities with ours, (e.g. sample size, type of patients and study period); even if we did not randomize patients we offer, as well, a comparison between CPAP alone and CPAP plus medical treatment. We used a different interface, able to provide a higher inspired oxygen fraction $\left(\mathrm{FiO}_{2}\right)$ and, likely, a better patient's tolerance.

\section{The helmet CPAP}

The device we use to deliver CPAP is a head helmet rather than a face mask: local experience justifies this choice, since at our institution we have been using helmet CPAP for more than 20 years in the ICUs, in the ER and in general wards; this might explain such a high rate of successful CPAP application, since most physicians and, particularly, nurses, had gained a good familiarity with helmet in the hospital.

In the specific pre hospital emergency care, a light, handy system is a must. Helmet CPAP [19] may appear cumbersome when compared to a traditional face mask circuitry [20] or to the Boussignac system [21]. Compared to a face mask however, the helmet does not require a critical fitting and is easily applied even by a minimally trained crew, causes only minimal discomfort to most patients [14]; it requires however a high enough gas flow to minimize pressure shifts along the respiratory cycle. With a $301 /$ min flow $\mathrm{CO}_{2}$ rebreathing is minimal $[16,22]$ and, since the helmet acts as a compliance chamber, the airway pressure is kept above PEEP throughout the respiratory cycle, provided that, as assessed by ALS crews, the gas flow through the expiratory valve is always present[16]. The spring-loaded valve used could have allowed some pressure swings in the helmet, however it does not increase the work of breathing [23], nor it affects the respiratory pattern, in comparison with an underwater seal valve (which could not have been used in the out-hospital setting anyway). Since it was the only one available at our institution when the study was 
conducted, we used a specific model of helmet (Sealong) whose use is less frequently reported in the literature than other models. Different (possibly better) results might have occurred by using more recent helmet models, subsequently developed and commercialized by other brands.

Kosowsky et al. applied mask CPAP by use of a powerful Venturi system at the expense of an $\mathrm{FiO}_{2}$ reduction; the system however was not portable and could be applied during ambulance transportation only. The Boussignac system tested by Templier et al. is indeed easily transportable but, again, a reduction in $\mathrm{FiO}_{2}$ can be anticipated [24, 25]. Finally, one should always take into account the rather high rate of patient's intolerance to the face mask (>10 \% in Kosowsky's study).

Also the Plaisance study employed a face mask with a powerful Venturi system, with a relatively low $\mathrm{FiO}_{2}$. No intolerance to face mask was reported[12].

Part of the benefits observed with CPAP in this study may be ascribed to the increase in $\mathrm{FiO}_{2}$ warranted by the helmet application. The device was indeed introduced to maximize $\mathrm{FiO}_{2}$ during hyperbaric treatment and it is entirely conceivable that $\mathrm{FiO}_{2}$ rose going from the bag mask to the helmet. Given its high compliance the head helmet guarantees a stable airway pressure also with a relatively low (30 1/min) fresh gas flow rate. This is not the case for the face mask, requiring a compromise between an elevated $\mathrm{FiO}_{2}$ and a good pneumatic performance. The demand for a very high fresh gas flow rate (Plaisance et al report 140 1/min), prevents the use of pure oxygen and mandates the use of a Venturi system with a consequent drop in $\mathrm{FiO}_{2}$. Although acceptable in the majority of the patients, this "trade-off" might become more problematic with the mostly hypoxic ones and we wonder if, with the use of an higher $\mathrm{FiO}_{2}$, some of the out-of-hospital intubations occurring in the Pleisance study might have been avoided.

\section{Efficacy of Helmet CPAP}

At variance with previous reports $[21,26]$, no patient required intubation or mechanical ventilation during transport. 
In-hospital intubation rate was nine out of 121 (7.4\%) homogeneously distributed between ALSdoc and ALSnurs groups. Among the intubated patients, six out of nine were diagnosed as non ACPE at ER discharge. This result compares favourably with the study by Kosowsky et al., reporting an in hospital intubation rate of 7 out of 19 (37\%). Intubation rate and overall mortality of the present study is in line with recent reports on the in-hospital $[4,7-10,27]$ and out-hospital [12] application of CPAP and Pressure Support Ventilation in ACPE. Oxygen saturation improved, respiratory rate, heart rate, and blood pressure decreased in both groups. Figure 1 shows that the reduction in blood pressure is proportional to the initial value. Moreover it can be observed that when SBP is near normal the application of CPAP does not lead to any significant hypotension. It could be speculated that CPAP, by improving oxygenation and decreasing the work of breathing, may indeed decrease the sympathetic tone. With this mechanism, CPAP may contribute to break the vicious circle that leads to pulmonary oedema. Moreover CPAP may actually improve cardiac function by decreasing left ventricular afterload through an increased intrathoracic pressure. This is, in turn, due to a direct increase in airway pressure and an indirect effect due to reduced negative pressure swings as a consequence of improved respiratory mechanics. Even in the subset of patients later diagnosed as non ACPE, helmet CPAP improved arterial oxygenation and did not influence negatively the hemodynamic status, confirming the safety of the procedure, although basic physiological knowledge suggests that the technique should be applied with caution in hypovolemic patients.

\section{Helmet CPAP with and without drug therapy (ALSdoc vs. ALSnurs)}

The comparison between the ALSdoc group and the ALSnurs group allows us to separate the effects of ventilatory support from those of pharmacological therapy. Somehow unexpectedly, CPAP with or without pharmacological therapy had strikingly similar effects on the clinical variables recorded until patients arrival in the ER, where all patients were treated with drugs. The two patient population appeared to be comparable except for a higher respiratory rate in the ALSnurs group. Despite a lower PEEP applied in ALSnurs (due to study design) both group 
responded similarly to helmet CPAP administration. In six patients of the ALSdoc group, a venous line was not available, nonetheless in this small group of patients helmet CPAP was extremely effective so that the absence of pharmacological treatment was not regretted. This observation actually prompted the application of CPAP by ALSnurs crews, not allowed to use drugs. Not surprisingly, given the possibility of applying an aggressive pharmacological therapy, the reduction in blood pressure was more pronounced in the ALSdoc than in the ALSnurs group (-14\% vs - 7 $\%$, see Table 3). However no relevant differences were seen in the improvement of the remaining clinical parameters and in the clinical outcomes. Although the relative short period of evaluation (on average around 30 minutes) might not have allowed the medical therapy to be fully effective, taken together these findings strongly suggest that the bulk of the observed effect are related to CPAP rather than to the pharmacological treatment. This is in keeping with the findings by Plaisance et al [12], demonstrating a more pronounced reduction in blood pressure with medical therapy as opposed to CPAP alone: however the comparison between the groups with an "early" and a "late" application of CPAP clearly shows that medical therapy alone (a condition which was not tested in our study) or as an adjunct to CPAP has a modest efficacy, if any, in improving respiratory conditions. If confirmed, this interpretation could actually suggest that an ALS crew staffed by nurses might manage out of hospital ACPE patients as effectively as a physician staffed crew until the necessary pharmacologic treatment is initiated.

\section{Conclusion}

Helmet CPAP was feasible, safe and effective in the pre-hospital treatment of presumed ACPE: it allowed prompt improvement in vital parameters both in association with pharmacological support and as a sole treatment. Out of hospital emergency intubation was avoided and in hospital intubation rate was low. The indication for pharmacologic treatment in ACPE is out of discussion. However, these observations suggest that helmet CPAP (along with drugs and especially before 
these can be administered) could be used as first line intervention in the pre hospital treatment of severe presumed ACPE.

\section{References}

1. Stiell IG, Spaite DW, Field B, Nesbitt LP, Munkley D, Maloney J, Dreyer J, Toohey LL, Campeau T, Dagnone E, Lyver M, Wells GA, (2007) Advanced life support for out-ofhospital respiratory distress. N Engl J Med 356: 2156-2164

2. Rasanen J, Heikkila J, Downs J, Nikki P, Vaisanen I, Viitanen A, (1985) Continuous positive airway pressure by face mask in acute cardiogenic pulmonary edema. Am J Cardiol 55: $296-300$

3. Bersten AD, Holt AW, Vedig AE, Skowronski GA, Baggoley CJ, (1991) Treatment of severe cardiogenic pulmonary edema with continuous positive airway pressure delivered by face mask. N Engl J Med 325: 1825-1830

4. Crane SD, Elliott MW, Gilligan P, Richards K, Gray AJ, (2004) Randomised controlled comparison of continuous positive airways pressure, bilevel non-invasive ventilation, and standard treatment in emergency department patients with acute cardiogenic pulmonary oedema. Emerg Med J 21: 155-161

5. Lin M, Yang YF, Chiang HT, Chang MS, Chiang BN, Cheitlin MD, (1995) Reappraisal of continuous positive airway pressure therapy in acute cardiogenic pulmonary edema. Shortterm results and long-term follow-up. Chest 107: 1379-1386

6. Masip J, Roque M, Sanchez B, Fernandez R, Subirana M, Exposito JA, (2005) Noninvasive ventilation in acute cardiogenic pulmonary edema: systematic review and meta-analysis. Jama 294: 3124-3130 
7. Nava S, Carbone G, DiBattista N, Bellone A, Baiardi P, Cosentini R, Marenco M, Giostra F, Borasi G, Groff P, (2003) Noninvasive ventilation in cardiogenic pulmonary edema: a multicenter randomized trial. Am J Respir Crit Care Med 168: 1432-1437

8. Pang D, Keenan SP, Cook DJ, Sibbald WJ, (1998) The effect of positive pressure airway support on mortality and the need for intubation in cardiogenic pulmonary edema: a systematic review. Chest 114: 1185-1192

9. Park M, Sangean MC, Volpe Mde S, Feltrim MI, Nozawa E, Leite PF, Passos Amato MB, Lorenzi-Filho G, (2004) Randomized, prospective trial of oxygen, continuous positive airway pressure, and bilevel positive airway pressure by face mask in acute cardiogenic pulmonary edema. Crit Care Med 32: 2407-2415

10. Peter JV, Moran JL, Phillips-Hughes J, Graham P, Bersten AD, (2006) Effect of noninvasive positive pressure ventilation (NIPPV) on mortality in patients with acute cardiogenic pulmonary oedema: a meta-analysis. Lancet 367: 1155-1163

11. Gray A, Goodacre S, Newby DE, Masson M, Sampson F, Nicholl J, (2008) Noninvasive ventilation in acute cardiogenic pulmonary edema. N Engl J Med 359: 142-151

12. Plaisance P, Pirracchio R, Berton C, Vicaut E, Payen D, (2007) A randomized study of outof-hospital continuous positive airway pressure for acute cardiogenic pulmonary oedema: physiological and clinical effects. Eur Heart J 28: 2895-2901

13. Mehta S, Hill NS, (2001) Noninvasive ventilation. Am J Respir Crit Care Med 163: 540-577

14. Principi T, Pantanetti S, Catani F, Elisei D, Gabbanelli V, Pelaia P, Leoni P, (2004) Noninvasive continuous positive airway pressure delivered by helmet in hematological malignancy patients with hypoxemic acute respiratory failure. Intensive Care Med 30: 147150. Epub 2003 Oct 2031.

15. Tonnelier JM, Prat G, Nowak E, Goetghebeur D, Renault A, Boles JM, L'Her E, (2003) Noninvasive continuous positive airway pressure ventilation using a new helmet interface: a case-control prospective pilot study. Intensive Care Med 29: 2077-2080. 
16. Patroniti N, Foti G, Manfio A, Coppo A, Bellani G, Pesenti A, (2003) Head helmet versus face mask for non-invasive continuous positive airway pressure: a physiological study. Intensive Care Med 29: 1680-1687

17. Delclaux C, L'Her E, Alberti C, Mancebo J, Abroug F, Conti G, Guerin C, Schortgen F, Lefort Y, Antonelli M, Lepage E, Lemaire F, Brochard L, (2000) Treatment of acute hypoxemic nonhypercapnic respiratory insufficiency with continuous positive airway pressure delivered by a face mask: A randomized controlled trial. Jama 284: 2352-2360

18. Squadrone V, Coha M, Cerutti E, Schellino MM, Biolino P, Occella P, Belloni G, Vilianis G, Fiore G, Cavallo F, Ranieri VM, (2005) Continuous positive airway pressure for treatment of postoperative hypoxemia: a randomized controlled trial. Jama 293: 589-595

19. Bellani G, Patroniti N, Greco M, Foti G, Pesenti A, (2008) The use of helmets to deliver non-invasive continuous positive airway pressure in hypoxemic acute respiratory failure. Minerva Anestesiol: Apr 18. [Epub ahead of print]

20. Kosowsky JM, Stephanides SL, Branson RD, Sayre MR, (2001) Prehospital use of continuous positive airway pressure (CPAP) for presumed pulmonary edema: a preliminary case series. Prehosp Emerg Care 5: 190-196

21. Templier F, Dolveck F, Baer M, Chauvin M, Fletcher D, (2003) 'Boussignac' continuous positive airway pressure system: practical use in a prehospital medical care unit. Eur $\mathbf{J}$ Emerg Med 10: 87-93

22. Taccone P, Hess D, Caironi P, Bigatello LM, (2004) Continuous positive airway pressure delivered with a "helmet": effects on carbon dioxide rebreathing. Crit Care Med 32: 20902096

23. Pelosi P, Chiumello D, Calvi E, Taccone P, Bottino N, Panigada M, Cadringher P, Gattinoni L, (2001) Effects of different continuous positive airway pressure devices and periodic hyperinflations on respiratory function. Crit Care Med 29: 1683-1689 
24. Villa F, Cereda M, Colombo E, Foti G, Pesenti A, (1999) Evaluation of four noninvasive CPAP systems. Intensive Care Med S6: A246

25. Templier F, Dolveck F, Baer M, Chauvin M, Fletcher D, (2003) [Laboratory testing measurement of FIO2 delivered by Boussignac CPAP system with an input of $100 \%$ oxygen]. Ann Fr Anesth Reanim 22: 103-107

26. Kallio T, Kuisma M, Alaspaa A, Rosenberg PH, (2003) The use of prehospital continuous positive airway pressure treatment in presumed acute severe pulmonary edema. Prehosp Emerg Care 7: 209-213

27. Domenighetti G, Gayer R, Gentilini R, (2002) Noninvasive pressure support ventilation in non-COPD patients with acute cardiogenic pulmonary edema and severe communityacquired pneumonia: acute effects and outcome. Intensive Care Med 28: 1226-1232 
Table 1. Inclusion and Exclusion Criteria.

\section{Inclusion Criteria}

- ALS Team availability

- Clinical Diagnosis of ACPE based on history and findings

- Pulse oximetry $<95 \%$ while breathing with $\mathrm{O}_{2}$ bag mask

\section{Exclusion Criteria}

- Need for immediate intubation

- Systolic Blood Pressure $<90 \mathrm{mmHg}$

- History of severe COPD

- History of spontaneous Pneumothorax

- Tracheotomy in place

List of Abbreviation: ALS Advanced Life Support. ACPE: Acute Pulmonary Cardiogenic Oedema, COPD: Chronic Obstructive Pulmonary Disease 
Table 2. Clinical Characteristics, Etiology of Acute Respiratory Failure (ARF), Outcome and Medical Treatment of Patients.

\begin{tabular}{|c|c|c|}
\hline & ALSdoc & ALSnurs \\
\hline $\mathbf{N}$ & 62 & 59 \\
\hline Age (years) & $78.5 \pm 7.8$ & $77.6 \pm 10.1$ \\
\hline Gender (Female/Male) & $37 / 25$ & $30 / 29$ \\
\hline \multicolumn{3}{|l|}{$\begin{array}{l}\text { OUTCOME } \\
\qquad \text { Intubation }\end{array}$} \\
\hline$\circ$ Out of Hospital & $\mathbf{0}$ & $\mathbf{0}$ \\
\hline$\circ$ In Hospital & 4 & 5 \\
\hline - ICU Admission & 9 & 13 \\
\hline - Overall Mortality & 11 & 9 \\
\hline \multicolumn{3}{|l|}{ Underlying cause of ARF } \\
\hline - $\mathrm{ACPE}$ & 54 & 50 \\
\hline - Other ARF & 8 & 9 \\
\hline ○ Pneumonia & 4 & 4 \\
\hline ○ Malignancies & 2 & 3 \\
\hline$\circ \quad A R D S$ & 2 & 2 \\
\hline \multicolumn{3}{|l|}{ Medical Treatment } \\
\hline - $C P A P$ & $62 / 62$ & $59 / 59$ \\
\hline - Drug Therapy & $56 / 62$ & $0 / 59$ \\
\hline O Furosemide & 50 & \\
\hline o i.v. Nitrates & 40 & \\
\hline ○ Morphine & 6 & \\
\hline$\circ \mathrm{Ca}^{++}$Antagonist & 2 & \\
\hline$\circ$ Digoxin & 1 & \\
\hline$\circ$ Aminophilline & 1 & \\
\hline
\end{tabular}

No statistically significant differences resulted between the two groups

List of Abbreviations. ALSdoc and ALSnurs: patients rescued by Advanced Life Support Teams staffed by physicians and by nurses respectively. ICU: Intensive Care Unit. ACPE: Acute 
Pulmonary Cardiogenic Oedema. ARDS: Acute Respiratory Distress Syndrome. CPAP: Continuous

Postive Airway Pressure 
Table 3. Respiratory and Hemodynamic Parameters.

\begin{tabular}{|c|c|c|c|c|c|c|}
\hline & \multicolumn{2}{|c|}{ Baseline } & \multicolumn{2}{|c|}{$C P A P$} & \multicolumn{2}{|c|}{ Variation (\%) } \\
\hline & ALSdoc & ALSnurs & ALSdoc & ALSnurs & ALSdoc & ALSnurs \\
\hline Arterial Oxygen Saturation (\%) & $79 \pm 12$ & $81 \pm 13$ & $97 \pm 3 *$ & $98 \pm 3 *$ & $19 \pm 12$ & $23 \pm 26$ \\
\hline $\begin{array}{l}\text { Respiratory Rate } \\
\text { (breaths per minute) }\end{array}$ & $26 \pm 4$ & $30 \pm 9 \S$ & $21 \pm 3^{*}$ & $22 \pm 8^{*}$ & $-21 \pm 15$ & $-24 \pm 17$ \\
\hline Wet Rales Score & $3.7 \pm 0.6$ & & $2.0 \pm 0.8 \#$ & & $-48 \pm 23$ & \\
\hline Systolic Blood Pressure (mmHg) & $175 \pm 49$ & $165 \pm 37$ & $145 \pm 28 *$ & $151 \pm 32 *$ & $-14 \pm 13 \S$ & $-7 \pm 15$ \\
\hline Heart Rate (beats per minute) & $112 \pm 23$ & $120.8 \pm 19.4$ & $105 \pm 19 *$ & $108 \pm 18^{*}$ & $-6 \pm 11$ & $-10 \pm 10$ \\
\hline $\begin{array}{l}\text { Heart Rate Pressure Product/100 } \\
\left(\mathrm{bpm}^{*} \mathrm{mmHg}\right)\end{array}$ & $191 \pm 56$ & $191 \pm 65$ & $151 \pm 38 *$ & $161 \pm 40 *$ & $-18 \pm 16$ & $-16 \pm 16$ \\
\hline Mean application time (minutes) & & & $34 \pm 6.6$ & $31.3 \pm 5.8$ & & \\
\hline
\end{tabular}

Main Physiologic parameters of the patients rescued by the Advanced Life Support Teams staffed by phyisicians (ALSdoc) and by nurses (ALS

Nurses), while breathing pure oxygen by face mask and during non-invasive Continuous Positive Airway Pressure (CPAP) Variation of each parameter are computed as $\left[\left(\mathrm{CPAP}_{\text {value }}-\mathrm{O} 2_{\text {value }}\right) / \mathrm{O} 2_{\text {value }}\right] * 100 * \mathrm{p}<0.01 \mathrm{O}_{2}$ Bag Mask vs. nCPAP, paired t-test and \# Wilcoxon-signed rank test, $\S$ $\mathrm{p}<0.01$ ALSdoc vs. ALSnurs unpaired t-test \# 


\section{Figure legend}

Figure 1: The figure shows the relative variation of the systolic blood pressure (SBP), induced by the application of non-invasive Continuous Positive Airway Pressure (CPAP) as a function of the initial value of SBP in patients rescued by Advanced Life Support teams staffed by physicians (empty symbols, dashed line) and by nurses (filled symbols solid line). Notice how the reduction in SBP increases with increasing baseline values of SBP (linear regression $p<0.01$ for both lines). Moreover, for the lowest value of baseline SBP (around $100 \mathrm{mmHg}$ ), the SBP does not decrease or, rather, increases, suggesting the safety of CPAP also in hypotensive patients. 